\title{
Are non-high-density lipoprotein fractions associated with pediatric metabolic syndrome? The CASPIAN-V study
}

\author{
Pooneh Angoorani ${ }^{1}$, Majid Khademian², Hanieh-Sadat Ejtahed ${ }^{3}$, Ramin Heshmat ${ }^{1}$, Mohammad Esmaeil Motlagh ${ }^{4}$, \\ Mahya Vafaeenia ${ }^{1}$, Gita Shafiee ${ }^{1}$, Armita Mahdivi-Gorabi ${ }^{1}$, Mostafa Qorbani ${ }^{5,6^{*}}$ and Roya Kelishadi ${ }^{*}$
}

\begin{abstract}
Background: Non-high-density lipoprotein cholesterol (non-HDL-C) is considered as a valuable predictor for dyslipidemia and subclinical atherosclerosis which can be an appropriate index for identifying individuals with metabolic syndrome (MetS).

Objective: To evaluate the association between non-HDL-C MetS and determine the optimal cut-points of non-HDL-C fractions for identifying MetS in Iranian children and adolescents.

Methods: This nationwide study was conducted in the framework of the fifth survey of a national schoolbased surveillance program on children and adolescents aged 7-18 years. MetS was defined by the Adult Treatment Panel III (ATP III) criteria modified for the pediatric age group. The analysis of receiver operating characteristic (ROC) curve was applied to determine the optimal cut-points of non-HDL-C, difference between non-HDL-C and LDL-C (Diff-C) and triglycerides (TG) to HDL-C ratio (TG/HDL-C) for the prediction of MetS.

Results: Overall, the study participants consisted of 3843 students (52.3\% boys) with mean ( \pm SD) age of 12.28 (3.1) years. The odds of high LDL-C, low HDL-C and MetS were increased in subjects with higher non-HDL-C, Diff-C and TG/HDL-C $(P<0.05)$. Non-HDL-C, Diff-C and TG/HDL-C cut-off points for predicting MetS were 120.5 mg/dl (sensitivity: 44\%, specificity: 73\%), 19.9 mg/dl (sensitivity: 85\%, specificity: 75\%) and 2.53 (sensitivity: 82\%, specificity: 79\%), respectively.

Conclusions: This study revealed a strong association between surrogates for serum lipid profile including non-HDL-C, TG/HDL-C and Diff-C and pediatric MetS. Our findings suggest that age- and gender-specific reference values of these markers were appropriate for both risk classification and long-term control of cardiovascular events in clinical assessments.
\end{abstract}

Keywords: Metabolic syndrome, Non-high-density lipoprotein cholesterol, Children, Adolescents

\section{Background}

The childhood obesity epidemic has increased dramatically over the past decades in parallel with an increase in prevalence of metabolic syndrome (MetS) in children and adolescents $[1,2]$. In Iran during the past decades, transition towards a sedentary lifestyle and unhealthy dietary

\footnotetext{
* Correspondence: mqorbani1379@yahoo.com; kelishadi@med.mui.ac.ir ${ }^{5}$ Non-communicable Diseases Research Center, Alborz University of Medical Sciences, Karaj, Iran

²Department of Pediatrics, Child Growth and Development Research Center, Research Institute for Primordial Prevention of Non-communicable Disease, Isfahan University of Medical Sciences, Hezar-Jarib Ave, Isfahan, Iran Full list of author information is available at the end of the article
}

pattern, has resulted in higher prevalence of MetS and its complications, especially among children and adolescents [3-5]. MetS in childhood can promote the risk of chronic diseases including diabetes mellitus, atherosclerosis and cardiovascular diseases (CVD), so early diagnosis and age-specific interventions have a substantial benefit in the prevention of its complications in adulthood [6].

Although commonly measured lipid parameters such as triglyceride (TG), high-density lipoprotein cholesterol (HDLC) and low-density lipoprotein cholesterol (LDL-C) can be acceptable alternatives for identifying individuals with CVD risks, recent studies show that non-conventional lipid profiles

(c) The Author(s). 2018 Open Access This article is distributed under the terms of the Creative Commons Attribution 4.0 International License (http://creativecommons.org/licenses/by/4.0/), which permits unrestricted use, distribution, and 
including non-high-density lipoprotein cholesterol (nonHDL-C), TG to HDL-C ratio (TG/HDL-C) or difference between non-HDL-C and LDL-C (Diff-C) are better than individual lipids in predicting cardiovascular events [7-9].

Non-HDL-C is an indicator of dyslipidemia calculated by subtracting high-density lipoprotein (HDL) cholesterol from total cholesterol (TC) and reflects the cholesterol in all atherogenic lipoprotein particles [10, 11]. The Adult Treatment Panel III of the National Cholesterol Education Program has considered non HDL-C as a recommended screening algorithm and American Diabetes Association and American College of Cardiology Foundation, suggested non-HDL-C as a better marker than LDL-C for predicting dyslipidemia and high-risk patients with CVD [12, 13]). Also in children, non-HDL-C has been shown to be a better indicator in anticipating dyslipidemia and subclinical atherosclerosis in adulthood compared to other lipid measures such as LDL-C [14, 15]. Because metabolic abnormalities are strongly associated with atherosclerosis, non-HDL-C can be an appropriate index for identifying individuals with MetS [16]). The strong association between non-HDL-C and MetS has been previously demonstrated [17-19]. However, the other evidences show that non-HDL-C levels varied in terms of sex, age group, and ethnic group [20]. Therefore, with respect to racial and genetic heterogeneities within and among populations, it seems necessary to determine the specific cut-points of non-HDL-C for each population. Thus, this study aims at evaluating the association between non-HDL cholesterol and MetS among Iranian children and adolescents and determining the optimal cut-off points of non-HDL-C fractions for recognition of MetS in these age groups. This study also determined the optimal cut-off points of TG/HDL-C ratio and Diff-C as important surrogate markers in cardiovascular risks.

\section{Methods}

\section{Study design and population}

The present study was conducted in the framework of the fifth survey of a national school-based surveillance survey entitled "Childhood and Adolescence Surveillance and Prevention of Adult Non-communicable Disease" (CASPIAN V) in 2015. Overall, 14,400 individuals without any history of chronic diseases aged 7-18 years participated in the survey. Sampling was conducted by multistage, stratified cluster sampling method from urban and rural areas of different cities in 30 provinces of Iran. Some students were randomly selected for biochemical test and fasting blood sample was collected. Protocol of this study has been explained in details previously [21].

The study was approved by the Research and Ethics Council of Isfahan University of Medical Sciences (code: 194049). After explanation of the study objectives and protocols, both written and verbal informed consent were obtained from all parents and pediatrics, respectively.

\section{Anthropometric and laboratory measurements}

Anthropometric measurements were performed by trained employee using calibrated instruments. Weight was measured to the nearest $0.1 \mathrm{~kg}$ while the subjects were minimally clothed without shoes. Height was measured in the standing position to the nearest $0.5 \mathrm{~cm}$ without shoes [22]. Body mass index (BMI) was calculated as weight $(\mathrm{kg})$ divided by square of height $\left(\mathrm{m}^{2}\right)$. Waist circumference (WC) was measured by a non-elastic tape to the nearest $0.1 \mathrm{~cm}$. Two measurements of blood pressure (BP) were done in the sitting position after $15 \mathrm{~min}$ of rest on the right arm using a standardized mercury sphygmomanometer. The first and fifth Korotkoff sounds were recorded as systolic blood pressure (SBP) and diastolic blood pressure (DBP), respectively.

Fasting blood samples were obtained from participants after a 12-14 h overnight fast. Fasting blood glucose (FBG), total cholesterol (TC), low density lipoprotein-cholesterol (LDL-C), high density lipoprotein-cholesterol (HDL-C), and triglycerides (TG) were measured enzymatically by Hitachi auto-analyzer (Tokyo, Japan). Non-HDL-C concentration was calculated by subtracting HDL-C concentration from total cholesterol concentration. Diff- $\mathrm{C}$ was defined as the difference between non-HDL-Cholesterol and LDLCholesterol. Atherogenic index was defined as the ratio between TG and HDL-C concentrations (TG/HDL-C) (19).

\section{Definition}

According to the modified Adult Treatment Panel III (ATP III) criteria modified for the pediatric age group, the diagnosis of MetS was established when three or more of the following components are present:

TG concentration of $150 \mathrm{mg} / \mathrm{dl}$ or greater; HDL-C concentration of $40 \mathrm{mg} / \mathrm{dL}$ or less; FBG concentration of $100 \mathrm{mg} / \mathrm{dl}$ or greater; abdominal obesity: waist to height ratio > 0.5; and either SBP or DBP greater than the 90th percentile for age, sex, and height [23].

Overweight and obesity among children were considered as a BMI between 85th and 95th percentile and BMI greater than 95th percentile for age and sex according to WHO criteria, respectively. High LDL was defined as LDL $>110 \mathrm{mg} / \mathrm{dl}$ and High TC was defined as $\mathrm{TC}>200 \mathrm{mg} / \mathrm{dl}$; Low HDL-C was defined as $\mathrm{HDL}<40 \mathrm{mg} / \mathrm{dl}$ except in boys 15-19 years old, that the cut-off was $<45 \mathrm{mg} / \mathrm{dl}$; elevated TG was defined as $\mathrm{TG}>100 \mathrm{mg} / \mathrm{dl}$.

\section{Statistical analysis}

Analyses were conducted using STATA version 11.0 (STATA Statistical Software: Release 11. STATA Corp LP. Package, College Station, TX, USA(. All variables 
were checked for normality and presented as the mean \pm standard deviation or number (percentage). The independent sample t-test was used to compare continuous variables and the Chi-square test was employed to compare proportions based on sex. Linear regression models were used to evaluate the association between nonHDL fractions and anthropometric and biochemical variables adjusting for potential confounders. Multivariate logistic regression analysis controlling for potential confounders including age, sex, living area, screen time, SES, physical activity and BMI was also performed to examine the association between non-HDL fractions and children with MetS and CVD risk factors. Data are presented as odds ratio (OR) with 95\% confidence interval $(\mathrm{CI})$.

The receiver operating characteristic (ROC) curve analysis was performed to determine the optimal cut-points of non-HDL fractions for the prediction of MetS (along with corresponding sensitivities and specificities). Data were also analyzed separately for different sex and age groups. The cut-off values were estimated by using the minimum value representing the maximum sum of sensitivity and specificity. The area under curve (AUC) shows the ability of non-HDL-C fraction of cut-off points to discriminate students with and without MetS. $P$-values less than 0.05 were considered statistically significant.

\section{Results}

The study participants consisted of 3843 students (52.3\% boys). General clinical and biochemical characteristics of the children and adolescents by gender are presented in Table 1. There were significant differences in mean age, waist, SBP, DBP, FBS, TC, LDL-C, and Non-HDL-C between girls and boys $(P<0.05)$. Boys had greater proportions of low HDL-C than girls (32.7\% VS 26.0\%, $P<0.001$ ). However, the prevalence of MetS and its other components was not significantly different between boys and girls.

The results of linear regression models between nonHDL-C fractions and anthropometric and biochemical variables are shown in Table 2. Non-HDL-C and TG/ HDL-C were positively associated with TG in three models $(P<0.05)$. HDL-C was negatively associated with Diff-C and TG/HDL-C $(P<0.05)$. LDL-C was positively associated with Non-HDL-C and Diff-C $(P<0.05)$. Moreover, TC and FBS were positively associated with Non-HDL-C, Diff-C and TG/HDL-C in all regression models $(P<0.05)$. Table 3 presents the odds ratios and 95\% confidence interval of obesity and MetS and its components associated with non-HDL-C fractions, using three logistic regression models. The odds of high TC, high LDL-C, MetS and low HDL-C were increased in pediatrics with higher non-HDL-C, Diff-C and TG/HDL-C $(P<$ 0.05). Theses associations remained significant after adjusting for age, sex, living area, socio-economic status,
Table 1 Characteristics of the study population: the CASPIAN-V study

\begin{tabular}{|c|c|c|c|c|}
\hline Variable & Boy & Girl & Total & $P$-value \\
\hline Age (year) ${ }^{a}$ & $12.39(3.1)$ & $12.17(3.1)$ & $12.28(3.1)$ & $<0.001$ \\
\hline BMI $(\mathrm{kg} / \mathrm{m} 2)^{\mathrm{a}}$ & $18.48(4.9)$ & $18.53(4.4)$ & $18.51(4.7)$ & 0.565 \\
\hline laist $(\mathrm{cm})^{\mathrm{a}}$ & $67.65(12.8)$ & $65.76(11.3)$ & $66.72(1.2)$ & $<0.001$ \\
\hline $\mathrm{BP}(\mathrm{mmHg})^{\mathrm{a}}$ & $99.55(13.4)$ & $98.77(12.8)$ & $99.17(13.0)$ & $<0.001$ \\
\hline $\mathrm{BP}(\mathrm{mmHg})^{\mathrm{a}}$ & $64.08(10.7)$ & $63.57(10.1)$ & $63.83(10.4)$ & 0.004 \\
\hline $\mathrm{G}(\mathrm{mg} / \mathrm{dl})^{\mathrm{a}}$ & $87.15(45.5)$ & $89.02(44.7)$ & $88.04(4.5)$ & 0.201 \\
\hline $\mathrm{DL}-\mathrm{C}(\mathrm{mg} / \mathrm{dl})^{\mathrm{a}}$ & $46.21(10.1)$ & $46.15(9.7)$ & $46.18(9.9)$ & 0.863 \\
\hline LDL-C (mg/dl) ${ }^{a}$ & $89.31(22.9)$ & $90.86(22.2)$ & $90.05(22.6)$ & 0.034 \\
\hline $\mathrm{TC}(\mathrm{mg} / \mathrm{dl})^{\mathrm{a}}$ & $152.95(28.0)$ & $154.82(26.6)$ & $153.84(27.4)$ & 0.035 \\
\hline $\mathrm{FBS}(\mathrm{mg} / \mathrm{dl})^{\mathrm{a}}$ & $92.06(12.9)$ & $91.19(11.1)$ & $91.64(12.1)$ & 0.027 \\
\hline Non-HDL-C (mg/dl) $)^{a}$ & $106.74(25.8)$ & $108.66(24.4)$ & $107.66(25.1)$ & 0.018 \\
\hline Diff-Chol $(\mathrm{mg} / \mathrm{dll})^{\mathrm{a}}$ & $17.43(9.1)$ & $17.80(8.9)$ & $17.60(9.0)$ & 0.201 \\
\hline $\mathrm{TG} / \mathrm{HDL}^{\mathrm{a}}$ & $2.03(1.3)$ & $2.08(1.3)$ & $2.05(1.3)$ & 0.265 \\
\hline MetS $S^{b}$ & $108(5.5)$ & $80(4.5)$ & $188(5.0)$ & 0.174 \\
\hline Low $\mathrm{HDL}^{\mathrm{b}}$ & $658(32.7)$ & $476(26.0)$ & $1134(29.5)$ & $<0.001$ \\
\hline High LDL ${ }^{\mathrm{b}}$ & 341 (16.9) & $333(18.2)$ & $674(17.5)$ & 0.310 \\
\hline High $T C^{b}$ & $100(5.0)$ & $89(4.9)$ & $189(4.9)$ & 0.878 \\
\hline Abdominal obesity ${ }^{b}$ & $1550(21.6)$ & $1422(20.5)$ & $2972(21.1)$ & 0.087 \\
\hline High $\mathrm{FBS}^{\mathrm{b}}$ & $96(4.8)$ & $65(3.8)$ & $161(4.2)$ & 0.060 \\
\hline High TG & $541(26.9)$ & $524(28.6)$ & $1065(27.7)$ & 0.228 \\
\hline High SBP & 210 & 3) & 438 & 0.255 \\
\hline High DBP ${ }^{b}$ & $746(10.5)$ & $704(10.2)$ & $1450(10.4)$ & 0.510 \\
\hline High $B P^{b}$ & 815 (11.5) & 789 (11.4) & $1604(11.5)$ & 0.45 \\
\hline
\end{tabular}

Data are presented as mean (SD)

${ }^{\mathrm{b}}$ Data are presented as number (percentage)

$S B P$ systolic blood pressure, $D B P$ Diastolic blood pressure, $B P$ blood pressure, $T G$ triglycerides, $F B G$ fasting blood glucose, $H D L-C$ high-density lipoproteincholesterol, TC total cholesterol, LDL-C low-density lipoprotein cholesterol low HDL: $<40 \mathrm{mg} / \mathrm{dL}$ (except in boys $15-19$ y old, that cut-off was $<45 \mathrm{mg} / \mathrm{dL}$ ); high LDL: > $110 \mathrm{mg} / \mathrm{dL}$; high TG: $100 \mathrm{mg} / \mathrm{dL}$; high TC: > $200 \mathrm{mg} / \mathrm{dL}$; elevated FBS > $100 \mathrm{mg} / \mathrm{dL}$; high blood pressure: > 90th (adjusted by age, sex, height)

screen time, physical activity and BMI $(P<0.05)$. There were no statistically significant associations between obesity, abdominal obesity and high blood pressure and non-HDL-C fractions. On the other hand, high FBS was significantly associated with Diff-C and TG/HDL-C (OR: 1.03, 95\% CI: $1.02-1.05$ and OR: 1.28 , 95\% CI: $1.17-1.40$, respectively). Furthermore, MetS was significantly associated with non- HDL-C, Diff-C and TG/HDL-C (OR: 1.01, 95\% CI: $1.01-1.02$, OR: $1.08,95 \%$ CI: $1.07-1.10$ and OR: $1.9,95 \%$ CI: $1.8-2.19$, respectively).

Results of the ROC analyses for gender and age groups are presented in Table 4. Non-HDL-C values for predicting MetS in boys, girls and the total pediatrics were considered to be 119.5 (sensitivity: $49 \%$, specificity: $73 \%$ and AUC: 61\%), 115.5 (sensitivity: 49\%, specificity: 64\% and AUC: $56 \%$ ) and 120.5 (sensitivity: 44\%, specificity: 73\% and AUC: $58 \%$ ), respectively. The Diff-C thresholds of 
Table 2 Associations between atherogenic indices and metabolic characteristics: the CASPIAN-V study

\begin{tabular}{lllllllllll}
\hline Variable & Model & ZBMI & WC & SBP & DBP & TG & HDL-C & LDL-C & TC & FBS \\
& & B (SE) & B (SE) & B (SE) & B (SE) & B (SE) & B (SE) & B (SE) & B (SE) & B (SE) \\
\hline Non-HDL-C (mg/dl) & Model I & $0.001(0.00)$ & $0.001(0.00)$ & $0.001(0.00)$ & $0.00(0.00)$ & $0.80(0.02)^{*}$ & $0.01(0.00)^{*}$ & $0.83(0.00)^{*}$ & $1.01(0.00)^{*}$ & $0.02(0.00)^{*}$ \\
& Model II & $0.001(0.00)$ & $0.01(0.00)$ & $0.01(0.00)$ & $0.00(0.00)$ & $0.79(0.02)^{*}$ & $0.01(0.00)$ & $0.84(0.00)^{*}$ & $1.01(0.00)^{*}$ & $0.02(0.00)^{*}$ \\
& Model III & - & - & $0.00(0.00)$ & $0.00(0.00)$ & $0.78(0.02)^{*}$ & $0.01(0.00)$ & $0.84(0.00)^{*}$ & $1.01(0.00)^{*}$ & $0.02(0.00)^{*}$ \\
Diff-C (mg/dl) & Model I & $0.001(0.00)^{*}$ & $0.03(0.02)$ & $0.03(0.02)$ & $0.02(0.01)$ & $0.23(0.04)^{*}$ & $-0.28(0.01)^{*}$ & $0.25(0.04)^{*}$ & $0.96(0.04)^{*}$ & $0.22(0.02)^{*}$ \\
& Model II & $0.001(0.00)^{*}$ & $0.04(0.02)$ & $0.03(0.02)$ & $0.02(0.01)$ & $0.20(0.03)^{*}$ & $-0.30(0.01)^{*}$ & $0.29(0.04)^{*}$ & $0.98(0.05)^{*}$ & $0.22(0.02)^{*}$ \\
& Model III & - & - & $0.01(0.02)$ & $0.01(0.01)$ & $0.19(0.02)^{*}$ & $-0.29(0.01)^{*}$ & $0.29(0.04)^{*}$ & $0.99(0.05)^{*}$ & $0.22(0.02)^{*}$ \\
Atherogenic index & Model I & $0.04(0.01)^{*}$ & $0.28(0.14)$ & $0.17(0.15)$ & $0.14(0.12)$ & $30.92(0.22)^{*}$ & $-3.88(0.10)^{*}$ & $0.10(0.27)$ & $2.40(0.32)^{*}$ & $1.30(0.14)^{*}$ \\
& Model II & $0.03(0.01)^{*}$ & $0.26(0.13)$ & $0.11(0.15)$ & $0.14(0.12)$ & $30.60(0.24)^{*}$ & $-3.99(0.11)^{*}$ & $0.26(0.29)$ & $2.39(0.35)^{*}$ & $1.30(0.15)^{*}$ \\
& Model III & - & - & $0.00(0.15)$ & $0.06(0.12)$ & $30.57(0.24)^{*}$ & $-3.98(0.11)^{*}$ & $0.26(0.29)$ & $2.39(0.35)^{*}$ & $1.31(0.15)^{*}$ \\
\hline
\end{tabular}

* Statistically significant, SBP systolic blood pressure, DBP diastolic blood pressure, BP blood pressure, TG triglycerides, $F B G$ fasting blood glucose, $H D L-C$ highdensity lipoprotein-cholesterol, $T C$ total cholesterol, $L D L-C$ low-density lipoprotein cholesterol, WC waist circumference, $z B M I z$ score of body mass index Non-HDL-C concentration was calculated by subtracting HDL-C value from total cholesterol concentration

Diff- $C$ was defined as the difference between total amount of non-HDL cholesterol and LDL-C (Diff- $C=[$ non-HDL- $C]-[L D L-C]$ ). Atherogenic index as the ratio between TG and HDL-C concentrations

Model I: crude model

Model II: adjusted for age, sex, living area, screen time, SES and physical activity

Model III: additionally adjusted for BMI except for zBMI and WC

Table 3 Odds ratio and 95\% confidence interval for cardiometabolic risk factors: the CASPIAN-V study

\begin{tabular}{|c|c|c|c|c|c|c|c|c|c|c|c|}
\hline \multirow[t]{2}{*}{ Variable } & \multirow[t]{2}{*}{ Model } & \multirow{2}{*}{$\begin{array}{l}\text { High TC } \\
\text { OR } \\
(95 \% \mathrm{Cl})\end{array}$} & \multirow{2}{*}{$\begin{array}{l}\text { High LDL } \\
\text { OR } \\
(95 \% \mathrm{Cl})\end{array}$} & \multirow{2}{*}{$\begin{array}{l}\text { MetS } \\
\text { OR } \\
(95 \% \mathrm{Cl}) \\
\end{array}$} & \multirow{2}{*}{$\begin{array}{l}\text { Low HDL } \\
\text { OR } \\
(95 \% \mathrm{Cl})\end{array}$} & \multirow{2}{*}{$\begin{array}{l}\text { Overweight } \\
\text { OR } \\
(95 \% \mathrm{Cl})\end{array}$} & \multirow{2}{*}{$\begin{array}{l}\text { Abdominal } \\
\text { obesity } \\
\text { OR } \\
(95 \% \mathrm{Cl})\end{array}$} & \multirow{2}{*}{$\begin{array}{l}\text { Obesity } \\
\text { OR } \\
(95 \% \mathrm{Cl})\end{array}$} & \multirow{2}{*}{$\begin{array}{l}\text { High FBS } \\
\text { OR } \\
(95 \% \mathrm{Cl})\end{array}$} & \multirow{2}{*}{$\begin{array}{l}\text { High TG } \\
\text { OR } \\
(95 \% \mathrm{Cl})\end{array}$} & \multirow{2}{*}{$\begin{array}{l}\text { High BP } \\
\text { OR } \\
(95 \% \mathrm{Cl})\end{array}$} \\
\hline & & & & & & & & & & & \\
\hline \multirow[t]{3}{*}{$\begin{array}{l}\text { Non-HDL-C } \\
(\mathrm{mg} / \mathrm{dl})\end{array}$} & $\begin{array}{l}\text { Model } \\
\text { I }\end{array}$ & $\begin{array}{l}1.19 \\
(1.17,1.22)^{*}\end{array}$ & $\begin{array}{l}1.19 \\
(1.17,1.20)^{*}\end{array}$ & $\begin{array}{l}1.01 \\
(1.00,1.01)^{*}\end{array}$ & $\begin{array}{l}0.99 \\
(0.99,0.99)^{*}\end{array}$ & $\begin{array}{l}1.00 \\
(0.99,1.00)\end{array}$ & $\begin{array}{l}1.00 \\
(0.99,1.00)\end{array}$ & $\begin{array}{l}1.00 \\
(0.99,1.00)\end{array}$ & $\begin{array}{l}1.00 \\
(1.00,1.01)\end{array}$ & $\begin{array}{l}1.03 \\
(1.03,1.03)^{*}\end{array}$ & $\begin{array}{l}0.99 \\
(0.99,1.00)\end{array}$ \\
\hline & $\begin{array}{l}\text { Model } \\
\|\end{array}$ & $\begin{array}{l}1.19 \\
(1.16,1.22)^{*}\end{array}$ & $\begin{array}{l}1.19 \\
(1.17,1.21)^{*}\end{array}$ & $\begin{array}{l}1.01 \\
(1.00,1.01)^{*}\end{array}$ & $\begin{array}{l}0.99 \\
(0.99,0.99)^{*}\end{array}$ & $\begin{array}{l}1.00 \\
(0.99,1.00)\end{array}$ & $\begin{array}{l}1.00 \\
(0.99,1.00)\end{array}$ & $\begin{array}{l}1.00 \\
(0.99,1.00)\end{array}$ & $\begin{array}{l}1.00 \\
(.99,1.01)\end{array}$ & $\begin{array}{l}1.03 \\
(1.02,1.03)^{*}\end{array}$ & $\begin{array}{l}0.99 \\
(0.99,1.00)\end{array}$ \\
\hline & $\begin{array}{l}\text { Model } \\
\text { III }\end{array}$ & $\begin{array}{l}1.19 \\
(1.16,1.22)^{*}\end{array}$ & $\begin{array}{l}1.19 \\
(1.17,1.21)^{*}\end{array}$ & $\begin{array}{l}1.01 \\
(1.00,1.01)^{*}\end{array}$ & $\begin{array}{l}0.99 \\
(0.99,0.99)^{*}\end{array}$ & - & - & - & $\begin{array}{l}1.00 \\
(.99,1.01)\end{array}$ & $\begin{array}{l}1.03 \\
(1.02,1.03)^{*}\end{array}$ & $\begin{array}{l}0.99 \\
(0.99,1.01)\end{array}$ \\
\hline \multirow[t]{3}{*}{$\begin{array}{l}\text { Diff-C } \\
(\mathrm{mg} / \mathrm{dl})\end{array}$} & $\begin{array}{l}\text { Model } \\
\text { l }\end{array}$ & $\begin{array}{l}1.07 \\
(1.06,1.08)^{*}\end{array}$ & $\begin{array}{l}1.02 \\
(1.01,1.02)^{*}\end{array}$ & $\begin{array}{l}1.07 \\
(1.06,1.09)^{*}\end{array}$ & $\begin{array}{l}1.04 \\
(1.03,1.05)^{*}\end{array}$ & $\begin{array}{l}1.01 \\
(1.00,1.02)^{*}\end{array}$ & $\begin{array}{l}1.00 \\
(0.99,1.01)\end{array}$ & $\begin{array}{l}1.00 \\
(0.99,1.01)\end{array}$ & $\begin{array}{l}1.04 \\
(1.02,1.05)^{*}\end{array}$ & $\begin{array}{l}1.02 \\
(1.01,1.02)^{*}\end{array}$ & $\begin{array}{l}1.00 \\
(0.98,1.01)\end{array}$ \\
\hline & $\begin{array}{l}\text { Model } \\
\|\end{array}$ & $\begin{array}{l}1.07 \\
(1.06,1.09)^{*}\end{array}$ & $\begin{array}{l}1.02 \\
(1.01,1.03)^{*}\end{array}$ & $\begin{array}{l}1.08 \\
(1.07,1.01)^{*}\end{array}$ & $\begin{array}{l}1.04 \\
(1.04,1.05)^{*}\end{array}$ & $\begin{array}{l}1.01 \\
(1.00,1.02)^{*}\end{array}$ & $\begin{array}{l}1.00 \\
(0.99,1.01)\end{array}$ & $\begin{array}{l}1.00 \\
(0.99,1.01)\end{array}$ & $\begin{array}{l}1.03 \\
(1.02,1.05)^{*}\end{array}$ & $\begin{array}{l}1.03 \\
(1.02,1.04)^{*}\end{array}$ & $\begin{array}{l}1.00 \\
(0.99,1.01)\end{array}$ \\
\hline & $\begin{array}{l}\text { Model } \\
\text { III }\end{array}$ & $\begin{array}{l}1.07 \\
(1.06,1.09)^{*}\end{array}$ & $\begin{array}{l}1.02 \\
(1.01,1.03)^{*}\end{array}$ & $\begin{array}{l}1.08 \\
(1.07,1.10)^{*}\end{array}$ & $\begin{array}{l}1.04 \\
(1.04,1.05)^{*}\end{array}$ & - & - & - & $\begin{array}{l}1.03 \\
(1.02,1.05)^{*}\end{array}$ & $\begin{array}{l}1.02 \\
(1.01,1.0\end{array}$ & $\begin{array}{l}1.00 \\
(0.98,1.01)\end{array}$ \\
\hline \multirow[t]{3}{*}{$\begin{array}{l}\text { Atherogenic } \\
\text { index }\end{array}$} & $\begin{array}{l}\text { Model } \\
\text { l }\end{array}$ & $\begin{array}{l}1.36 \\
(1.26,1.46)^{*}\end{array}$ & $\begin{array}{l}1.02 \\
(0.96,1.08)\end{array}$ & $\begin{array}{l}1.8 \\
(1.7,2.00)^{*}\end{array}$ & $\begin{array}{l}2.43 \\
(2.25,2.63)^{*}\end{array}$ & $\begin{array}{l}1.08 \\
(1.00,1.16)^{*}\end{array}$ & $\begin{array}{l}1.00 \\
(0.95,1.06)\end{array}$ & $\begin{array}{l}1.03 \\
(0.95,1.10)\end{array}$ & $\begin{array}{l}1.29 \\
(1.19,1.40)^{*}\end{array}$ & $\begin{array}{l}38.69 \\
(29.85,50.14)^{*}\end{array}$ & $\begin{array}{l}1.00 \\
(0.92,1.08)\end{array}$ \\
\hline & $\begin{array}{l}\text { Model } \\
\|\end{array}$ & $\begin{array}{l}1.34 \\
(1.24,1.46)^{*}\end{array}$ & $\begin{array}{l}1.03 \\
(0.96,1.10)\end{array}$ & $\begin{array}{l}1.9 \\
(1.7,2.14)^{*}\end{array}$ & $\begin{array}{l}2.51 \\
(2.30,2.73)^{*}\end{array}$ & $\begin{array}{l}1.07 \\
(0.98,1.15)\end{array}$ & $\begin{array}{l}1.01 \\
(0.95,1.08)\end{array}$ & $\begin{array}{l}1.03 \\
(0.95,1.12)\end{array}$ & $\begin{array}{l}1.29 \\
(1.18,1.41)^{*}\end{array}$ & $\begin{array}{l}40.33 \\
(30.45,53.43)^{*}\end{array}$ & $\begin{array}{l}1.02 \\
(0.94,1.11)\end{array}$ \\
\hline & $\begin{array}{l}\text { Model } \\
\text { III }\end{array}$ & $\begin{array}{l}1.35 \\
(1.24,1.47)^{*}\end{array}$ & $\begin{array}{l}1.03 \\
(0.96,1.10)\end{array}$ & $\begin{array}{l}1.9 \\
(1.8,2.19)^{*}\end{array}$ & $\begin{array}{l}2.50 \\
(2.30,2.72)^{*}\end{array}$ & - & - & - & $\begin{array}{l}1.28 \\
(1.17,1.40)^{*}\end{array}$ & $\begin{array}{l}40.26 \\
(30.36,53.40)^{*}\end{array}$ & $\begin{array}{l}1.00 \\
(0.92,1.09)\end{array}$ \\
\hline
\end{tabular}

* Statistically significant, BP blood pressure, TG triglycerides, FBG fasting blood glucose, HDL-C high-density lipoprotein-cholesterol, TC total cholesterol, LDL-C lowdensity lipoprotein cholesterol;

overweight: BMl; 85th-95th; obesity, BMI > 95th; low HDL: < 40 mg/dL (except in boys 15-19 y old, that cut-off was < 45 mg/dL); high LDL: > 110 mg/dL; high TG: $100 \mathrm{mg} / \mathrm{dL}$; high TC: $>200 \mathrm{mg} / \mathrm{dL}$; elevated FBS > $100 \mathrm{mg} / \mathrm{dL}$; high blood pressure: > 90th (adjusted by age, sex, height); Non-HDL-C concentration was calculated by subtracting HDL-C value from total cholesterol concentration. Diff-C was defined as the difference between total amount of non-HDL cholesterol and LDL-C (Diff-C $=[$ non-HDL-C]-[LDL-C]). Atherogenic index as the ratio between TG and HDL-C concentrations

Model 1: crude model

Model 2: adjusted for age, sex, living area, screen time, SES and physical activity

Model3: additionally adjusted for BMI except for overweight, obesity and abdominal obesity 
Table 4 Receiver operator curve for Non-High-Density lipoprotein fractions for identifying children with MetS

\begin{tabular}{|c|c|c|c|c|c|}
\hline Variable & Age-sex group & Cut-off points $(95 \%$ Cl) & Sensitivity $(95 \%$ Cl) & Specificity $(95 \%$ Cl) & $\overline{A \cup C}$ \\
\hline \multirow[t]{16}{*}{ Non-HDL-C (mg/dl) } & $7-10 y$ & & & & \\
\hline & Boy & $\begin{array}{l}163 \\
(96.29,227.70)\end{array}$ & $\begin{array}{l}0.69 \\
(0.585,0.807)\end{array}$ & $\begin{array}{l}0.73 \\
(0.48,0.97)\end{array}$ & 0.71 \\
\hline & Girl & $\begin{array}{l}104.5 \\
(88.21,119.78)\end{array}$ & $\begin{array}{l}0.71 \\
(0.47,0.95)\end{array}$ & $\begin{array}{l}0.50 \\
(0.28,0.70)\end{array}$ & 0.60 \\
\hline & Total & $\begin{array}{l}104.5 \\
(73.99,134.00)\end{array}$ & $\begin{array}{l}0.60 \\
(0.28,0.91)\end{array}$ & $\begin{array}{l}0.50 \\
(0.14,0.85)\end{array}$ & 0.55 \\
\hline & $11-14 y$ & & & & \\
\hline & Boy & $\begin{array}{l}119.5 \\
(107.00,130.99)\end{array}$ & $\begin{array}{l}0.54 \\
(0.34,0.74)\end{array}$ & $\begin{array}{l}0.73 \\
(0.59,0.85)\end{array}$ & 0.64 \\
\hline & Girl & $\begin{array}{l}116.5 \\
(77.57,154.42)\end{array}$ & $\begin{array}{l}0.49 \\
(0.10,0.86)\end{array}$ & $\begin{array}{l}0.65 \\
(0.21,1.07)\end{array}$ & 0.57 \\
\hline & Total & $\begin{array}{l}116.5 \\
(111.38,120.61)\end{array}$ & $\begin{array}{l}0.53 \\
(0.37,0.68)\end{array}$ & $\begin{array}{l}0.67 \\
(0.57,0.76)\end{array}$ & 0.60 \\
\hline & $15-18 y$ & & & & \\
\hline & Boy & $\begin{array}{l}101.5 \\
(81.67,120.32)\end{array}$ & $\begin{array}{l}0.91 \\
(0.66,1.16)\end{array}$ & $\begin{array}{l}0.51 \\
(0.27,0.73)\end{array}$ & 0.71 \\
\hline & Girl & $\begin{array}{l}115.5 \\
(75.49,154.50)\end{array}$ & $\begin{array}{l}0.54 \\
(0.07,1.00)\end{array}$ & $\begin{array}{l}0.64 \\
(0.14,1.14)\end{array}$ & 0.59 \\
\hline & Total & $\begin{array}{l}104.5 \\
(83.66,124.33)\end{array}$ & $\begin{array}{l}0.79 \\
(0.52,1.06)\end{array}$ & $\begin{array}{l}0.52 \\
(0.25,0.78)\end{array}$ & 0.65 \\
\hline & $7-18 y$ & & & & \\
\hline & Boy & $\begin{array}{l}119.5 \\
(103.37,134.62)\end{array}$ & $\begin{array}{l}0.49 \\
(0.26,0.71)\end{array}$ & $\begin{array}{l}0.73 \\
(0.50,0.95)\end{array}$ & 0.61 \\
\hline & Girl & $\begin{array}{l}115.5 \\
(88.58,141.41)\end{array}$ & $\begin{array}{l}0.49 \\
(0.18,0.78)\end{array}$ & $\begin{array}{l}0.64 \\
(0.25,1.01)\end{array}$ & 0.56 \\
\hline & Total & $\begin{array}{l}120.5 \\
(105.33,134.66)\end{array}$ & $\begin{array}{l}0.44 \\
(0.22,0.65)\end{array}$ & $\begin{array}{l}0.73 \\
(0.52,0.93)\end{array}$ & 0.58 \\
\hline \multirow[t]{14}{*}{ Diff-C (mg/dl) } & $7-10 y$ & & & & \\
\hline & Boy & $\begin{array}{l}19.9 \\
(17.47,22.12)\end{array}$ & $\begin{array}{l}0.78 \\
(0.60,0.95)\end{array}$ & $\begin{array}{l}0.78 \\
(0.72,0.83)\end{array}$ & 0.78 \\
\hline & Girl & $\begin{array}{l}20.7 \\
(15.94,25.25)\end{array}$ & $\begin{array}{l}0.89 \\
(0.75,1.00)\end{array}$ & $\begin{array}{l}0.78 \\
(0.66,0.89)\end{array}$ & 0.84 \\
\hline & Total & $\begin{array}{l}19.9 \\
(18.88,20.71)\end{array}$ & $\begin{array}{l}0.84 \\
(0.73,0.93)\end{array}$ & $\begin{array}{l}0.77 \\
(0.72,0.81)\end{array}$ & 0.80 \\
\hline & $11-14 y$ & & & & \\
\hline & Boy & $\begin{array}{l}19.9 \\
(18.26,21.33)\end{array}$ & $\begin{array}{l}0.83 \\
(0.71,0.93)\end{array}$ & $\begin{array}{l}0.77 \\
(0.72,0.81)\end{array}$ & 0.80 \\
\hline & Girl & $\begin{array}{l}19.9 \\
(18.18,21.41)\end{array}$ & $\begin{array}{l}0.82 \\
(0.69,0.94)\end{array}$ & $\begin{array}{l}0.73 \\
(0.64,0.82)\end{array}$ & 0.78 \\
\hline & Total & $\begin{array}{l}19.9 \\
(18.41,21.18)\end{array}$ & $\begin{array}{l}0.82 \\
(0.74,0.90)\end{array}$ & $\begin{array}{l}0.75 \\
(0.71,0.78)\end{array}$ & 0.79 \\
\hline & $15-18 y$ & & & & \\
\hline & Boy & $\begin{array}{l}19.9 \\
(18.05,21.54)\end{array}$ & $\begin{array}{l}0.91 \\
(0.83,0.99)\end{array}$ & $\begin{array}{l}0.74 \\
(0.67,0.80)\end{array}$ & 0.83 \\
\hline & Girl & $\begin{array}{l}20.5 \\
(15.45,25.34)\end{array}$ & $\begin{array}{l}0.92 \\
(0.75,1.08)\end{array}$ & $\begin{array}{l}0.76 \\
(0.68,0.84)\end{array}$ & 0.84 \\
\hline & Total & $\begin{array}{l}19.9 \\
(19.16,20.43)\end{array}$ & $\begin{array}{l}0.92 \\
(0.84,0.99)\end{array}$ & $\begin{array}{l}0.74 \\
(0.70,0.77)\end{array}$ & 0.83 \\
\hline & $7-18 y$ & & & & \\
\hline & Boy & $\begin{array}{l}19.9 \\
(19.26,20.33)\end{array}$ & $\begin{array}{l}0.84 \\
(0.76,0.91)\end{array}$ & $\begin{array}{l}0.76 \\
(0.73,0.79)\end{array}$ & 0.80 \\
\hline
\end{tabular}


Table 4 Receiver operator curve for Non-High-Density lipoprotein fractions for identifying children with MetS (Continued)

\begin{tabular}{|c|c|c|c|c|c|}
\hline Variable & Age-sex group & Cut-off points (95\% Cl) & Sensitivity $(95 \% \mathrm{Cl})$ & Specificity $(95 \% \mathrm{Cl})$ & AUC \\
\hline & Girl & $\begin{array}{l}19.9 \\
(19.37,20.22)\end{array}$ & $\begin{array}{l}0.86 \\
(0.78,0.93)\end{array}$ & $\begin{array}{l}0.74 \\
(0.70,0.78)\end{array}$ & 0.80 \\
\hline & Total & $\begin{array}{l}19.9 \\
(19.76,19.83)\end{array}$ & $\begin{array}{l}0.85 \\
(0.79,0.90)\end{array}$ & $\begin{array}{l}0.75 \\
(0.74,0.76)\end{array}$ & 0.80 \\
\hline \multirow[t]{16}{*}{ Atherogenic index } & $7-10 y$ & & & & \\
\hline & Boy & $\begin{array}{l}2.53 \\
(1.83,3.23)\end{array}$ & $\begin{array}{l}0.70 \\
(0.52,0.88)\end{array}$ & $\begin{array}{l}0.83 \\
(0.70,0.95)\end{array}$ & 0.77 \\
\hline & Girl & $\begin{array}{l}2.57 \\
(2.03,3.10)\end{array}$ & $\begin{array}{l}0.93 \\
(0.83,1.02)\end{array}$ & $\begin{array}{l}0.81 \\
(0.69,0.92)\end{array}$ & 0.87 \\
\hline & Total & $\begin{array}{l}2.53 \\
(2.10,2.96)\end{array}$ & $\begin{array}{l}0.82 \\
(0.72,0.90)\end{array}$ & $\begin{array}{l}0.81 \\
(0.71,0.91)\end{array}$ & 0.82 \\
\hline & $11-14 y$ & & & & \\
\hline & Boy & $\begin{array}{l}2.58 \\
(2.37,2.78)\end{array}$ & $\begin{array}{l}0.80 \\
(0.70,0.90)\end{array}$ & $\begin{array}{l}0.82 \\
(0.78,0.86)\end{array}$ & 0.81 \\
\hline & Girl & $\begin{array}{l}2.54 \\
(1.96,3.11)\end{array}$ & $\begin{array}{l}0.79 \\
(0.65,0.93)\end{array}$ & $\begin{array}{l}0.77 \\
(0.61,0.93)\end{array}$ & 0.78 \\
\hline & Total & $\begin{array}{l}2.54 \\
(2.25,2.83)\end{array}$ & $\begin{array}{l}0.80 \\
(0.70,0.89)\end{array}$ & $\begin{array}{l}0.80 \\
(0.72,0.87)\end{array}$ & 0.80 \\
\hline & $15-18 y$ & & & & \\
\hline & Boy & $\begin{array}{l}2.48 \\
(2.04,2.91)\end{array}$ & $\begin{array}{l}0.89 \\
(0.75,1.01)\end{array}$ & $\begin{array}{l}0.75 \\
(0.65,0.83)\end{array}$ & 0.82 \\
\hline & Girl & $\begin{array}{l}2.73 \\
(2.24,3.22)\end{array}$ & $\begin{array}{l}0.92 \\
(0.79,1.05)\end{array}$ & $\begin{array}{l}0.82 \\
(0.75,0.88)\end{array}$ & 0.87 \\
\hline & Total & $\begin{array}{l}2.48 \\
(2.17,2.78)\end{array}$ & $\begin{array}{l}0.90 \\
(0.79,99)\end{array}$ & $\begin{array}{l}0.76 \\
(0.69,0.82)\end{array}$ & 0.83 \\
\hline & $7-18 y$ & & & & \\
\hline & Boy & $\begin{array}{l}2.53 \\
(2.35,2.71)\end{array}$ & $\begin{array}{l}0.80 \\
(0.71,0.88)\end{array}$ & $\begin{array}{l}0.80 \\
(0.76,0.83)\end{array}$ & 0.80 \\
\hline & Girl & $\begin{array}{l}2.54 \\
(2.19,2.89)\end{array}$ & $\begin{array}{l}0.86 \\
(0.77,0.94)\end{array}$ & $\begin{array}{l}0.79 \\
(0.71,0.86)\end{array}$ & 0.83 \\
\hline & Total & $\begin{array}{l}2.53 \\
(2.41,2.65)\end{array}$ & $\begin{array}{l}0.82 \\
(0.76,0.88)\end{array}$ & $\begin{array}{l}0.79 \\
(0.76,0.82)\end{array}$ & 0.81 \\
\hline
\end{tabular}

$\mathrm{Cl}$ confidence interval, AUC area under curve, shown as percentage

Non-HDL-C concentration was calculated by subtracting HDL-C value from total cholesterol concentration. Diff- $C$ was defined as the difference between total amount of non-HDL cholesterol and LDL-C (Diff- $C=[$ non-HDL-C]-[LDL-C]). Atherogenic index as the ratio between TG and HDL-C concentrations

19.9 predicted MetS in boys (sensitivity: $84 \%$, specificity: 76\% and AUC: $80 \%$ ), girls (sensitivity: 86\%, specificity: $74 \%$ and AUC: $80 \%$ ) and the total pediatrics (sensitivity: 85\%, specificity: $75 \%$ and AUC: $80 \%$ ). TG/HDL-C thresholds of 2.53 (sensitivity: $80 \%$, specificity: $80 \%$ and AUC: $80 \%$ ), 2.54 (sensitivity: $86 \%$, specificity: $79 \%$ and AUC: $83 \%$ ) and 2.53 (sensitivity: $82 \%$, specificity: $79 \%$ and AUC: $81 \%$ ) were considered in order to predict MetS in boys, girls and the total pediatrics, respectively.

\section{Discussion}

In the current study, we presented the age- and sex-stratified cut off values of serum lipid profiles including non-HDL-C, TG/HDL-C and Diff-C for predicting MetS in Iranian children and adolescents from a national school-based surveillance survey. The thresholds were determined $120 \mathrm{mg} / \mathrm{dl}, 2.53$ and $19.9 \mathrm{mg} / \mathrm{dl}$ for non-HDL-C,
TG/HDL-C ratio and Diff-C, respectively in the total participants. The findings demonstrated that the higher values of non-HDL-C, TG/HDL-C and Diff-C were associated with increased risk of MetS in this age group.

Several studies have recommended the use of nonHDL-C as a valuable predictor for dyslipidemia and cardiovascular risks $[19,24,25]$. Because dyslipidemia is a hallmark of metabolic syndrome and prevalence of dyslipidemia in Iran is high [26-28], this study evaluated the relation between non-HDL-C and MetS. The reference values for non-conventional lipid profiles such as non-HDL-C for recognition of MetS in children and adolescents have been determined in few studies. In a survey on US children and adolescents aged 12-19 years, non-HDL-C was strongly associated with MetS and its thresholds was determined $120 \mathrm{mg} / \mathrm{dl}$ and $145 \mathrm{mg} / \mathrm{dl}$ to indicate borderline and high MetS risk, respectively [29]. 
Consistent with US study, this study showed that non-HDL-C above $120 \mathrm{mg} / \mathrm{dl}$ was associated with MetS in Iranian children and adolescents. The Expert Panel on Integrated Guidelines for Cardiovascular Health and Risk Reduction in Children and Adolescents has suggested the use of the non-fasting non-HDL-C level and non-HDL-C $\geq 145 \mathrm{mg} / \mathrm{dl}$ as a universal screening level for dyslipidemia in children and adolescents up to 19 years [30]. Shim et al. presented the age and genderspecific reference values for non-HDL-C and the TG/ HDL-C in Korean children and adolescents aged 1019 years as a valuable information for individualized interpretations of lipid profiles and interventions as well as for strategies to prevent and manage childhood and adolescent dyslipidemia [31]. Saito et al. in a study on Japanese obese boys aged $12.0 \pm 2.6$ years with MetS demonstrated that higher non-HDL-C levels is associated with increased risk for the development of atherosclerosis [32].

Few studies have reported reference values for TG/ HDL-C ratio and Diff- $C$ in children and adolescents. In confirming our findings, the TG/HDL-C ratio was shown as an independent predictor of atherosclerosis in survey conducted on US adolescents and young adults [33]. In a large Italian study, the TG/HDL-C ratio is presented as a simple and effective method for diagnosis CVD and dyslipidemia in children and adolescents [34]. Even it was documented that Diff-C fraction and TG/HDL-C ratio are able to predict MetS more accurately than total non-HDL-C $[19,34]$ and the TG/HDL-C ratio proved a better index than HOMA-IR in screening for MetS in obese children and adolescents [35]. However, non-HDL-C is more popular than TG/HDL-C ratio and Diff-C worldwide [36]. Overall, the consistent findings regarding the association between these lipid profile surrogates and cardiovascular risk in different population with different ethnicity, culture, food pattern and lifestyle, reveals the utility and importance of these indices in the managements of MetS in children and adolescents across the globe.

Non-HDL-C is suggested as an appropriate method for screening dyslipidemia and cardiovascular risk in the pediatric setting because overnight fasting is not necessary before the measurement of non-HDL-C [30]. Furthermore, since TG variability can lead to significant changes in LDL-C evaluation in children and adolescents, nonHDL-C level can be more reliable than LDL-C concentration in this age group [37]. In fact, Non-HDL-C reflects the amounts of the TG-rich lipoprotein content of several atherogenic particles, including LDL-C, intermediatedensity lipoprotein cholesterol (IDL-C), very-low-density lipoprotein cholesterol (VLDL-C) and chylomicron remnants. It has been indicated that IDL and small VLDL values, but not LDL value, associate with increased risk of atherosclerosis [38, 39]. The atherogenic effect of small TG-rich lipoproteins is due to their association with clotting and the fibrinolytic pathway through producing foam cells [40]. Moreover, non-HDL-C indicates an estimate of all ApoB-containing lipoproteins, all of which have the potential to take cholesterol into the arterial wall and result in atherosclerotic lesions. Therefore, non-HDL-C can mirrors atherogenic risk not captured by LDL-C measurement alone, especially in the case of elevated TG [41].

In this study, among five components of MetS, high TG and low HDL-C were significantly associated with nonHDL-C. Non-HDL-C contains atherogenic lipoproteins, including LDL-C, IDL-C, VLDL-C and correlates highly with serum TG levels. Because serum TG is mainly carried in lipid-rich particles including VLDL, fasting serum TG levels strongly associates with VLDL-C concentration. Besides, high serum TG levels often are accompanied by abnormal levels of small particles of LDL and low HDL-C concentration [42]. However, TG and non-HDL-C have extremely different biochemical specifications and metabolic mechanisms; non-HDL-C mostly via atherogenic process, and TG mostly via insulin resistance, are associated with CVD risk [43].

For the first time, this study presents a sex and agestratified cut-off values of non-HDL-C, TG/HDL-C and Diff-C for predicting MetS in a nationally representative sample of Iranian children and adolescents aged 718 years and evaluates the association between nonHDL-C concentration and MetS in multivariable logistic regression analyses based on standard protocols. Nevertheless, this study has some limitations which have to be pointed out. First, the cross-sectional design of this study did not let us to obtain any causality between nonHDL-C concentration and MetS. Second, we had no data regarding the prevalence of atherosclerosis or CVD in these children and adolescents so we could not show inferences on the association between non-HDL-C and these events as definite end points. Further studies are suggested to assess the association between lipid profile surrogates in children and adolescents and cardiovascular status of parents. Moreover, performing specific cardiac physical examinations including ecocolordoppler with intima- media thickness (IMT) measurement could provide more accurate information to clarify these associations.

\section{Conclusion}

According to racial and genetic heterogeneities between populations, we determined the specific cut-points of serum lipid profiles including non-HDL-C, TG/HDL-C ratio and Diff- $\mathrm{C}$ for predicting MetS in 7-18 year-olds Iranian children and adolescents. The robust association between these lipid profile surrogates and both current MetS and future atherosclerosis supported the use of these parameters as appropriate methods in both risk classification and long-term control of CVD risk in the clinical assessments. 


\section{Abbreviations}

ATP III: Adult treatment panel III; BMI: Body mass index; BP: Blood pressure; CASPIAN: Childhood and adolescence surveillance and prevention of adult non-communicable disease; Cl: Confidence interval; CVD: Cardiovascular diseases; DBP: Diastolic blood pressure; Diff-C: Difference between non-highdensity lipoprotein cholesterol and low-density lipoprotein cholesterol; FBG: Fasting blood glucose; HDL-C: High-density lipoprotein cholesterol; LDL-C: Low-density lipoprotein cholesterol; MetS: Metabolic syndrome; NonHDL-C: Non-high-density lipoprotein cholesterol; OR: Odds ratio; ROC: Receiver operating characteristic; SBP: Systolic blood pressure; SD: Standard deviation; TG: Triglycerides; TG/HDL-C: Triglycerides to high-density lipoprotein cholesterol ratio; WC: Waist circumference

\section{Acknowledgements}

The authors are thankful of all participants and large team working on this project in different provinces.

\section{Funding}

This research did not receive any specific grant from funding agencies in the public, commercial, or not-for-profit sectors.

\section{Availability of data and materials}

The datasets used in this study are available from the corresponding author on reasonable request.

\section{Authors' contributions}

Concept: H-SE, MQ, Design: RH, MQ, RK, Data Collection or Processing: RH, MEM, MV, AM-G, GS, PA, Analysis or Interpretation: H-SE, MQ, PA, Literature Search: H-SE, MQ, MK, Writing: H-SE, PA, RK. All authors read and approved the final manuscript.

\section{Ethics approval and consent to participate}

The study was approved by the Research and Ethics Council of Isfahan University of Medical Sciences (code: 194049). After explanation of the study objectives and protocols, both written and verbal informed consent were obtained from all parents and pediatrics, respectively.

\section{Consent for publication}

All authors agree to publish this work.

\section{Competing interests}

No potential conflicts of interest relevant to this article were reported by any of the authors. None of the funding sources played a role in the design, collection, analysis or interpretation of the data or in the decision to submit the manuscript for publication. The authors declare that they have no competing interests.

\section{Publisher's Note}

Springer Nature remains neutral with regard to jurisdictional claims in published maps and institutional affiliations.

\section{Author details}

${ }^{1}$ Chronic Diseases Research Center, Endocrinology and Metabolism Population Sciences Institute, Tehran University of Medical Sciences, Tehran, Iran. ${ }^{2}$ Department of Pediatrics, Child Growth and Development Research Center, Research Institute for Primordial Prevention of Non-communicable Disease, Isfahan University of Medical Sciences, Hezar-Jarib Ave, Isfahan, Iran. ${ }^{3}$ Obesity and Eating Habits Research Center, Endocrinology and Metabolism Clinical Sciences Institute, Tehran University of Medical Sciences, Tehran, Iran. ${ }^{4}$ Department of Pediatrics, Ahvaz Jundishapur University of Medical Sciences, Ahvaz, Iran. ${ }^{5}$ Non-communicable Diseases Research Center, Alborz University of Medical Sciences, Karaj, Iran. ${ }^{6}$ Endocrinology and Metabolism Research Center, Endocrinology and Metabolism Clinical Sciences Institute, Tehran University of Medical Sciences, Tehran, Iran.

Received: 25 August 2018 Accepted: 21 October 2018 Published online: 14 November 2018

\section{References}

1. Ejtahed H-S, Qorbani M, Motlagh ME, Angoorani P, Hasani-Ranjbar S, Ziaodini $\mathrm{H}$, et al. Association of anthropometric indices with continuous metabolic syndrome in children and adolescents: the CASPIAN-V study. Eat Weight Disord. 2017:1-8. https://doi.org/10.1007/s40519-017-0455-0.

2. A systematic review on the prevalence of metabolic syndrome in Iranian children and adolescents.

3. Ejtahed H-S, Bahadoran Z, Mirmiran P, Azizi F. Sugar-sweetened beverage consumption is associated with metabolic syndrome in Iranian adults: Tehran lipid and glucose study. Endocrinol Metab. 2015;30:334-42.

4. Angoorani P, Ejtahed H-S, Mirmiran P, Mirzaei S, Azizi F. Dietary consumption of advanced glycation end products and risk of metabolic syndrome. Int J Food Sci Nutr. 2016;67:170-6.

5. Angoorani P, Heshmat R, Ejtahed H-S, Motlagh ME, Ziaodini H, Taheri M, et al. The association of parental obesity with physical activity and sedentary behaviors of their children: the CASPIAN-V study. In: J Pediatr (Rio J); 2017. https://doi.org/10.1016/j.jped.2017.06.024.

6. Day C. Metabolic syndrome, or what you will: definitions and epidemiology. Diab Vasc Dis Res. 2007:4:32-8.

7. Onat A, Can G, Kaya H, Hergenc G. "Atherogenic index of plasma" (log10 triglyceride/high-density lipoprotein-cholesterol) predicts high blood pressure, diabetes, and vascular events. J Clin Lipidol. 2010;4:89-98.

8. Gasevic D, Frohlich J, Mancini GJ, Lear SA. Clinical usefulness of lipid ratios to identify men and women with metabolic syndrome: a cross-sectional study. Lipid Health Dis. 2014;13:159.

9. Cobbaert C, Jukema JW, Zwinderman AH, Withagen AJ, Lindemans J, Bruschke AV. Modulation of lipoprotein (a) Atherogenicity by high density lipoprotein cholesterol levels in middle-aged men with symptomatic coronary artery disease and Normal to moderately elevated serum cholesterol fn1. J Am Coll Cardiol. 1997:30:1491-9.

10. Cui Y, Blumenthal RS, Flaws JA, Whiteman MK, Langenberg P, Bachorik PS, et al. Non-high-density lipoprotein cholesterol level as a predictor of cardiovascular disease mortality. Arch Intern Med. 2001;161:1413-9.

11. Tanabe N, Iso H, Okada K, Nakamura Y, Harada A, Ohashi Y, et al. Serum total and non-high-density lipoprotein cholesterol and the risk prediction of cardiovascular events. Circ J. 2010;74(7):1346-56.

12. Grundy SM, Cleeman JI, Merz CNB, Brewer HB, Clark LT, Hunninghake DB, et al. Implications of recent clinical trials for the national cholesterol education program adult treatment panel III guidelines. Circulation. 2004; 110:227-39

13. Brunzell JD, Davidson M, Furberg CD, Goldberg RB, Howard BV, Stein JH, et al. Lipoprotein management in patients with cardiometabolic risk: consensus statement from the American Diabetes Association and the American College of Cardiology Foundation. Diabetes Care. 2008;31:811-22.

14. Srinivasan SR, Frontini MG, Xu J, Berenson GS. Utility of childhood nonhigh-density lipoprotein cholesterol levels in predicting adult dyslipidemia and other cardiovascular risks: the Bogalusa heart study. Pediatrics. 2006;118:201-6.

15. Frontini MG, Srinivasan SR, Xu J, Tang R, Bond MG, Berenson GS. Usefulness of childhood non-high density lipoprotein cholesterol levels versus other lipoprotein measures in predicting adult subclinical atherosclerosis: the Bogalusa heart study. Pediatrics. 2008;121(5):924-9.

16. Tsai S-S, Lin Y-S, Chen S-T, Chu P-H. Metabolic syndrome positively correlates with the risks of atherosclerosis and diabetes in a Chinese population. Eur J Case Rep Intern Med. 2018. https://doi.org/10.1016/j.ejim. 2018.04.009 [Epub ahead of print].

17. Kim SW, Jee JH, Kim HJ, Jin S-M, Suh S, Bae JC, et al. Non-HDL-cholesterol/HDLcholesterol is a better predictor of metabolic syndrome and insulin resistance than apolipoprotein B/apolipoprotein A1. Intern J Cardiol. 2013;168:2678-83.

18. Choi BH, Hye JS, Cha CK, Ahn Y. Non-HDL cholesterol as a risk factor of metabolic syndrome in Korean women. Korean J Obes. 2007;16(3):102-10.

19. Ghodsi S, Meysamie A, Abbasi M, Ghalehtaki R, Esteghamati A, Malekzadeh MM, et al. Non-high-density lipoprotein fractions are strongly associated with the presence of metabolic syndrome independent of obesity and diabetes: a population-based study among Iranian adults. J Diabetes Metab Disord. 2017;16:25.

20. Srinivasan SR, Myers L, Berenson GS. Distribution and correlates of nonhigh-density lipoprotein cholesterol in children: the Bogalusa Heart Study. Pediatrics. 2002;110:e29-e.

21. Motlagh ME, Ziaodini H, Qorbani M, Taheri M, Aminaei T, Goodarzi A, et al. Methodology and early findings of the fifth survey of childhood and adolescence surveillance and prevention of adult noncommunicable disease: the CASPIAN-V study. Int J Prev Med. 2017;8. https://doi.org/10. 4103/2008-7802.198915. 
22. Status WP. The use and interpretation of anthropometry. Geneva CH: WHO 1995, technical report; 1995. p. 854.

23. Zimmet P, Alberti G, Kaufman F, Tajima N, Silink M, Arslanian S, et al. The metabolic syndrome in children and adolescents. Lancet. 2007;369:2059-61.

24. Homma S, Troxclair DA, Zieske AW, Malcom GT, Strong JP. Histological changes and risk factor associations in type 2 atherosclerotic lesions (fatty streaks) in young adults. Atherosclerosis. 2011;219:184-90.

25. Frontini MG, Srinivasan SR, Xu J-H, Tang R, Bond MG, Berenson G. Utility of non-high-density lipoprotein cholesterol versus other lipoprotein measures in detecting subclinical atherosclerosis in young adults (the Bogalusa heart study). Am J Cardiol. 2007;100:64-8.

26. Prevalence of dyslipidemia in iran: a systematic review and meta-analysis study.

27. Prevalence of dyslipidemia in Iranian children and adolescents: A systematic review.

28. Mean serum lipid levels in Iranian adult populations: a systematic review and meta-analysis.

29. Li C, Ford ES, McBride PE, Kwiterovich PO, McCrindle BW, Gidding SS. Non-high-density lipoprotein cholesterol concentration is associated with the metabolic syndrome among US youth aged 12-19 years. J Pediatr. 2011;158:201-7.

30. FOR EPOIG, CHILDREN RRI. Expert panel on integrated guidelines for cardiovascular health and risk reduction in children and adolescents: summary report. Pediatrics. 2011;128(Suppl 5):S213.

31. Shim YS, Baek JW, Kang MJ, Oh YJ, Yang S. II TH. Reference values for the triglyceride to high-density lipoprotein cholesterol ratio and nonhigh-density lipoprotein cholesterol in Korean children and adolescents: the Korean National Health and nutrition examination surveys 2007-2013. J Atheroscler Thromb. 2016;23:1334-44.

32. Saito E, Okada T, Abe Y, Kazama M, Yonezawa R, Kuromori Y, et al. Nonhigh-density lipoprotein cholesterol levels in Japanese obese boys with metabolic syndrome. J Atheroscler Thromb. 2016;23:105-11.

33. Urbina EM, Khoury PR, McCoy CE, Dolan LM, Daniels SR, Kimball TR. Triglyceride to HDL-C ratio and increased arterial stiffness in children, adolescents, and young adults. Pediatrics. 2013. https://doi.org/10.1542/peds.2012-1726.

34. Di Bonito P, Valerio G, Grugni G, Licenziati M, Maffeis C, Manco M, et al. CARdiometabolic risk factors in overweight and obese children in ITALY (CARITALY) study group: comparison of non-HDL-cholesterol versus triglycerides-to-HDL-cholesterol ratio in relation to cardiometabolic risk factors and preclinical organ damage in overweight/obese children: the CARITALY study. Nutr Metab Cardiovasc Dis. 2015;25:489-94.

35. Liang J, Fu J, Jiang Y, Dong G, Wang X, Wu W. TriGlycerides and high-density lipoprotein cholesterol ratio compared with homeostasis model assessment insulin resistance indexes in screening for metabolic syndrome in the chinese obese children: a cross section study. BMC Pediatr. 2015:15:138.

36. Abe Y. Difference in serum non-high-density lipoprotein cholesterol levels in terms of sex, age, and physique in children and adolescents. J Atheroscler Thromb. 2016;23:1311-2.

37. Gidding SS, Stone NJ, Bookstein LC, Laskarzewski PM, Stein EA. Month-tomonth variability of lipids, lipoproteins, and apolipoproteins and the impact of acute infection in adolescents. J Pediatr. 1998:133:242-6.

38. Mack WJ, Krauss RM, Hodis HN. Lipoprotein subclasses in the monitored atherosclerosis regression study (MARS): treatment effects and relation to coronary angiographic progression. Arterioscler Thromb Vasc Biol. 1996; 16:697-704.

39. Hodis HN, Mack WJ, Dunn M, Liu C-R, Liu C-H, Selzer RH, et al. Intermediatedensity lipoproteins and progression of carotid arterial wall intima-media thickness. Circulation. 1997:95(8):2022-6

40. Hodis HN. Triglyceride-rich lipoprotein remnant particles and risk of atherosclerosis. Am Heart Assoc. 1999:99(22):2852-4.

41. Ballantyne CM, Andrews TC, Hsia JA, Kramer JH, Shear C. Correlation of nonhigh-density lipoprotein cholesterol with apolipoprotein B: effect of 5 hydroxymethylglutaryl coenzyme a reductase inhibitors on non-highdensity lipoprotein cholesterol levels. Am J Cardiol. 2001;88:265-9.

42. Grundy SM. Hypertriglyceridemia, atherogenic dyslipidemia, and the metabolic syndrome. Am J Cardiol. 1998;81:18B-25B.

43. Li C, Ford ES, Meng Y-X, Mokdad AH, Reaven GM. Does the association of the triglyceride to high-density lipoprotein cholesterol ratio with fasting serum insulin differ by race/ethnicity? Cardiovasc Diabetol. 2008;7:4

\section{Ready to submit your research? Choose BMC and benefit from:}

- fast, convenient online submission

- thorough peer review by experienced researchers in your field

- rapid publication on acceptance

- support for research data, including large and complex data types

- gold Open Access which fosters wider collaboration and increased citations

- maximum visibility for your research: over $100 \mathrm{M}$ website views per year

At $\mathrm{BMC}$, research is always in progress.

Learn more biomedcentral.com/submissions 